\title{
DIRECT ESTIMATION OF NUTATION COEFFICIENTS FROM VLBI DATA
}

\author{
W. E. Himwich and E. J. Harder \\ Interferometrics Inc. \\ 8150 Leesburg Pike, Suite 1400 \\ Vienna, VA 22180
}

\begin{abstract}
Previous analyses of nutation angle errors from Mark III VLBI data have been made by estimating offset corrections to the instantaneous nutation angles from single-day observing sessions. Adjustments to the coefficients of the nutation model were then estimated from the time series of offset corrections. In this paper we estimate the corrections to the nutation series coefficients directly from the multi-year ensemble of data. The resulting corrections have smaller uncertainties than corrections estimated from the daily offsets because the direct solution has many fewer degrees of freedom. We have forced the corrections to occur at the periods of the dominant terms of the IAU 1980 nutation series instead of allowing the pole orientation to vary freely on each day. Results from daily offset adjustment and direct nutation series adjustment from nearly seven years of data agree at the level of the combined one-sigma uncertainties, 0.1 milliarcseconds. The amplitude of the free core nutation was also estimated from the daily offset data; no significant amplitude was observed.
\end{abstract}

\section{INTRODUCTION}

Discrepancies between the IAU 1980 nutation series and the observed nutation angles were first reported in Herring et al. (1986). Herring used Mark III Very Long Baseline Interferometry (VLBI) data to estimate offsets in longitude and obliquity from the IAU 1980 series for each day of data. These offsets represented a time series of corrections to the a priori series. Corrections to the coefficients of the nutation series were estimated by fitting the offsets to the dominant terms of the nutation series with periods of one year or less. The only significant corrections were to the annual and semi-annual terms. Gwinn et al. (1986) interpreted the corrections to the annual terms as being caused by an error in the free core nutation (FCN) period used in the theory. Gwinn calculated a new FCN period based on the observations and predicted corrections to the 6798.4-day terms.

We present here the results of a different type of analysis of Mark III VLBI data for the recovery of nutation corrections. The 
corrections to the coefficients of the IAU 1980 series are estimated directly. This removes any errors caused by averaging nutation angle corrections over a single day of data, a small effect. It also takes fully into account the correlations of the corrections on different days and between the corrections on the same day. These correlations are not normally taken into account for analyses using daily offsets because of the computational complexity involved in utilizing them.

The most significant effect of estimating the coefficients directly is the reduction of the number of degrees of freedom in the solution. The nutation corrections are constrained to occur with power at the periods of the adjusted coefficients. This prevents each day from having an arbitrary pole orientation and reduces the uncertainties of the corrections to the nutation coefficients. Forcing the nutation corrections to occur only at specific periods also prevents the estimated pole position on a given day from being contaminated by correlations of the nutation corrections with other parameters, notably clock and atmospheric model parameters.

The principal advantage of using the daily offset technique is that it allows arbitrary precession and nutation model errors to be corrected for without a priori knowledge of their form. With direct estimation of the coefficients, the terms with significant corrections must be known beforehand. The use of daily offsets and direct estimation are complementary techniques which have different strengths. Direct estimation produces more precise estimates of the corrections if their form is already known.

\section{METHODS AND DATA}

In order to compare the two analysis techniques, a data span of 7 years was analyzed using both. We estimated corrections for the in-phase and out-of-phase components of the 6798.4-day, 182.6-day, 121.7-day, and 13.7-day terms for both longitude and obliquity. We omitted the 3399.2-day term since no correction is expected for that term and we cannot reliably extract corrections to both the 3399.2-day and 6798.4-day terms with the existing span of data, about 2400 days.

We used all of the fixed observatory Mark III VLBI data taken by the NASA Crustal Dynamics Project (CDP) through July 17, 1986 and all of the data taken by the POLARIS and International Radio Interferometric Survey (IRIS) networks through July 21, 1986. There are 425 individual sessions of which about 80 percent are from POLARIS/IRIS. The CDP, however, contributes almost half of the 155,000 observations.

We analyzed the data to estimate corrections to the nutation coefficients in a single large least-squares solution. We solved for more than 10,000 parameters including the 20 corrections to the nutation series. The coordinates of the sources were estimated simultaneously with the nutation corrections. For each individual session an independent set of station coordinates was estimated to prevent the results from being biased by any unmodeled station motion.

A second solution was made to estimate daily offsets. All of the 
features of this solution were identical to the coefficient adjustment solution except how nutation corrections were estimated. In this case we estimated a longitude and an obliquity offset for each session except one. The day on which offsets were not adjusted is the 'reference day'. Not adjusting the offsets on one day serves to fix the orientation of the celestial reference frame relative to the terrestrial reference frame. Both frames are being estimated, but the relative orientation between them is fixed.

The estimated daily offsets were further processed to separately fit each time series, one in obliquity, the other in longitude, to the same terms of the nutation series we adjusted in the direct solution. A bias and rate were estimated for each series as well. A bias corrects for a non-zero error in the a priori nutation angles on the reference day. A rate removes the effect of possible longer term errors in the nutation and precession models.

\section{RESULTS}

The estimated corrections to the IAU 1980 nutation coefficients from the direct solution and from daily offsets are shown in Table I. The difference between the estimates from the two techniques is at or below the combined one-sigma uncertainty, about 0.1 milliarcseconds, for all of the terms except the 6798.4-day terms. The 6798.4-day terms are not expected to agree at this level because we solved for biases and rates when analyzing the daily offsets but not in the direct solution. The corrections for the 6798.4-day terms estimated from the daily offsets are strongly correlated with the biases and rates. The corrections for the 6798.4-day terms estimated directly may have aliased an error in the precession model, since no attempt was made to compensate for such an error. The shorter period, well-determined corrections agree very we11.

The one-sigma uncertainties for the direct estimation technique are the formal one-sigma uncertainties from the overall solution scaled to make the reduced Chi-square unity and then scaled by a factor of two. The choice of a factor of two is discussed below. The reduced Chi-square was 0.98 before scaling.

The calculation of the one-sigma uncertainties for the corrections estimated from the daily offsets is more involved. The formal onesigma uncertainties for daily offsets were scaled to make the reduced Chi-square unity. The reduced Chi-square was 0.96 before scaling. The formal one-sigma uncertainties from the estimation of the nutation corrections were then scaled to make the reduced Chi-square of that solution unity. The reduced Chi-square of the second fit was about 2.8 before scaling. These errors were then multiplied by two.

As has been described, the uncertainties from both analysis techniques have been inflated by a factor of two. This was done because there maybe systematic errors which have not been properly considered. The most significant error is probably improper modeling of the tropospheric delay. The choice of a factor of two will not be justified in detail here. However, many investigators have studied the 
TABLE I. Corrections to the IAU 1980 Nutation Series from Direct Estimation and from Daily offsets. This table compares the corrections estimated from the two different techniques. The data used to estimate the corrections span seven years. The daily offset corrections for the 6798.4-day terms may have large errors since they were estimated simultaneously with a bias and rate. The direct estimates of the 6798.4-day terms may have aliased an error in the precession model since no attempt was made to compensate for such an error. The longitude values have been scaled by the sine of the obliquity. Corrections to terms of only these five periods were calculated for this comparison.

\begin{tabular}{|c|c|c|c|c|}
\hline \multirow[b]{2}{*}{$\begin{array}{l}\text { Period } \\
\text { (days) }\end{array}$} & \multicolumn{2}{|c|}{$\begin{array}{l}\text { Corrections from Direct } \\
\text { Obliquity }\end{array}$} & \multicolumn{2}{|l|}{ Estimation } \\
\hline & $\begin{array}{l}\text { in-phase } \\
\text { (mas) }\end{array}$ & $\begin{array}{l}\text { out-of-phase } \\
\text { (mas) }\end{array}$ & $\begin{array}{l}\text { in-phase } \\
\text { (mas) }\end{array}$ & $\begin{array}{l}\text { out-of-phase } \\
\text { (mas) }\end{array}$ \\
\hline 6798.4 & $1.591 \pm 0.092$ & $0.792 \pm 0.240$ & $-1.717 \pm 0.266$ & $-1.825 \pm 0.116$ \\
\hline & $57 \pm 0.045$ & $0.082 \pm 0.04$ & $769 \pm 0.050$ & $87 \pm 0.050$ \\
\hline & $05 \pm \quad "$ & $-0.410 \pm \quad "$ & $0.438 \pm \quad "$ & $13 \pm \quad "$ \\
\hline & $5 \pm$ & $-0.042 \pm$ & $022 \pm$ & $74 \pm$ \\
\hline 13.7 & $99 \pm$ & $0.246 \pm$ & $517 \pm$ & $061 \pm$ \\
\hline & $\begin{array}{r}\text { Correct } \\
\mathrm{Ob}\end{array}$ & $\begin{array}{l}\text { Estimated from } \\
\text { dity }\end{array}$ & $\begin{array}{l}\text { Daily Offsets } \\
\text { Longitude }\end{array}$ & \\
\hline Period & $\begin{array}{l}\text { in-phase } \\
\text { (mas) }\end{array}$ & $\begin{array}{l}\text { out-of-phase } \\
\text { (mas) }\end{array}$ & $\begin{array}{l}\text { in-phase } \\
\text { (mas) }\end{array}$ & $\begin{array}{l}\text { out-of-phase } \\
\text { (mas) }\end{array}$ \\
\hline & $5 \pm 3.13$ & $1.310 \pm 0.76$ & $460 \pm 0.783$ & \pm 3.177 \\
\hline 36 & $1.911 \pm 0.110$ & $0.072 \pm 0.110$ & $801 \pm 0.115$ & $0 \pm 0.115$ \\
\hline & $7 \pm \quad "$ & $-0.469 \pm \quad "$ & $30 \pm$ & $1 \pm$ \\
\hline & $14 \pm$ & $-0.052 \pm$ & $03 \pm$ & $27 \pm$ \\
\hline 13.7 & $0.209 \pm$ & $0.156 \pm$ & $-0.438 \pm$ & $0.036 \pm$ \\
\hline
\end{tabular}

effects of systematic errors on the data, see Herring et al. (1986) for example. A factor of two to three increase in the formal uncertainties is usually appropriate. For our purposes the most important consideration is that the uncertainties in both types of estimation have been calculated consistently so that they may be directly compared.

\section{DISCUSSION}

The agreement of the two types of analysis is very good, at or below the one-sigma level generally. This good agreement between the directly estimated nutation corrections and the corrections estimated from daily offsets gives support to the daily offset technique. The approximations made by the daily offset technique (that orientation of the pole is constant over a single day, that the correlations between the offsets are insignificant, and that the effect of correlations of other parameters with the offsets is insignificant) are justified as reasonable. The direct estimation technique does not make those approximations and produces very comparable results. 
TABLE II. Estimates of the free core nutation amplitude and corrections to the IAU 1980 Nutation Series estimated from daily offsets. The amplitude of the free core nutation (FCN) amplitude was estimated using a period of 433.5 Julian days. The phase used to calculate the FCN terms increases with time and is zero at epoch J2000.0. The corrections for the 6798.4-day terms may have large errors since they were estimated simultaneously with a bias and rate. The longitude values have been scaled by the sine of obliquity.

\begin{tabular}{|c|c|c|c|c|c|}
\hline & \multicolumn{2}{|c|}{ obliquity } & \multicolumn{3}{|c|}{ Longitude (scaled) } \\
\hline $\begin{array}{l}\text { Period } \\
\text { (days) }\end{array}$ & $\begin{array}{l}\text { in-phase } \\
\text { (mas) }\end{array}$ & $\begin{array}{c}\text { out-of-phase } \\
\text { (mas) }\end{array}$ & $\begin{array}{l}\text { in-phase } \\
\text { (mas) }\end{array}$ & out - & $\begin{array}{l}\text {-of-phase } \\
\text { (mas) }\end{array}$ \\
\hline 6798.4 & $1.477 \pm 3.166$ & $1.352 \pm 0.772$ & $-3.426 \pm 0.737$ & 5.682 & $2 \pm 3.003$ \\
\hline 433.5 & $-0.245 \pm 0.140$ & $0.087 \pm 0.140$ & $-0.498 \pm 0.135$ & -0.078 & $8 \pm 0.135$ \\
\hline 365.3 & $1.797 \pm \quad "$ & $-0.078 \pm \quad "$ & $1.681 \pm \quad "$ & 0.555 & $5 \pm \quad 1$ \\
\hline 182.6 & $-0.312 \pm 0.110$ & $-0.494 \pm 0.110$ & $0.512 \pm 0.105$ & -0.290 & $0 \pm 0.105$ \\
\hline 121.7 & $-0.013 \pm \quad "$ & $-0.047 \pm \quad "$ & $-0.093 \pm$ & -0.062 & $2 \pm \quad "$ \\
\hline 31.8 & $0.050 \pm$ & $0.041 \pm$ & $0.069 \pm$ & -0.004 & $4 \pm$ \\
\hline 27.6 & $-0.141 \pm$ & $0.120 \pm$ & $-0.068 \pm$ & 0.160 & $0 \pm$ \\
\hline 13.7 & $0.213 \pm$ & $0.155 \pm$ & $-0.421 \pm$ & 0.073 & $3 \pm$ \\
\hline 9.1 & $-0.016 \pm$ & $0.080 \pm$ & $-0.034 \pm$ & -0.014 & $4 \pm$ \\
\hline
\end{tabular}

The agreement of direct estimates with Herring et al. (1986) for terms with periods of one year or less is excellent. The out-of-phase annual term in obliquity reported here is somewhat smaller than that reported by Herring. The direct estimates of the corrections to 6798.4-day terms do not agree very well, except for the in-phase obliquity correction, with the predictions of Gwinn et al. (1986). The significance of these corrections is doubtful given the short span of data available. The agreement of the in-phase obliquity correction must be treated as fortuitous for the time being.

The uncertainties of the corrections estimated from the daily offsets in this paper are smaller than Herring's. This is due to the larger data set analyzed here. The uncertainties from direct estimation are less than half the size of those from daily offsets estimated from the same data set. This is indicative of the increased precision inherent in direct estimation.

There is some interest in seeing if there is a significant detection of the free core nutation (FCN). To test this, the daily offsets were fit to a larger set of components. This fit included the terms of the original five periods plus the 31.8-day, 27.6-day, 9.1day, and FCN terms. The FCN terms were estimated using sine and cosine components with a period of 433.5 Julian days. The phase used to calculate the FCN terms increases with time and is zero at epoch J2000.0. The 13.6-day terms were not estimated since its separation from the 13.7-day terms would be poor for this short span of data.

The solution using this expanded set of parameters had a reduced Chi-square of 2.3, about twenty percent smaller than the previous solution from offsets. The results are shown in Table II. There are no terms which have significant corrections other than the 6798.4-day, 
433.5-day, 365.3-day, 182.6-day, and 13.7-day terms. A well determined estimate of the correction to the 6798.4-day terms will not be possible until we have a longer span of data. The 13.7-day term seems to have a significant correction. In this solution it is only three sigma detection, although in the direct solution it was four to ten sigma.

For the FCN, two derived parameters, the prograde and retrograde amplitudes, are of the most interest. Expressions for the real and imaginary components of both can be found in Herring et al. (1986). The amplitudes are the root-sum-square of the components. The FCN terms have a retrograde amplitude of $0.38 \pm 0.14$ mas and a prograde amplitude of $0.13 \pm 0.14$ mas. This is almost a 3 -sigma detection for the retrograde amplitude. The retrograde amplitude is larger than the prograde, as would be expected, but how much this is caused by the retrograde annual correction being larger than the prograde is unclear. A longer time span of data will be required to more clearly separate the FCN amplitude from the annual correction.

In order to improve the determination of the longer period terms, we plan to investigate using the Mark I geodetic VLBI data to increase the time span of the solution by about 7 years to a total of 14 years. Unfortunately, these data are of lower precision than the Mark III data. In addition there is no ionospheric calibration available. The effect of not having the ionospheric calibration must be investigated in detail before the Mark I data can be included.

\section{CONCLUSION}

Correcting the 1980 IAU nutation coefficients by using daily offsets and by direct correction of the nutation series have been shown to yield comparable results. Direct estimation of the corrections to the amplitudes produces higher precision estimates than can be obtained from daily offsets. As more data becomes available a clearer detection of the FCN, if it exists, will become possible. Work is planned to improve the separation of the 6798.4-day correction from errors in the precession constant.

\section{ACKNOWLEDGEMENTS}

We would like to thank C. Yoder and C. Gwinn for sharing their understanding of nutation theory with us.

\section{REFERENCES}

Gwinn C. R., T. A. Herring, and I. I. Shapiro, 'Geodesy by Radio Interferometry: Studies of the Forced Nutation of the Earth, 2 . Interpretation', J. Geophys. Res. 91, 4745-4754, 1986.

Herring, T. A., C. R. Gwinn, and I. I. Shapiro, 'Geodesy by Radio Interferometry: Studies of the Forced Nutation of the Earth, 1. Data Analysis', J. Geophys. Res. 91, 4755-4765, 1986. 


\section{DISCUSSION}

Dickey: In both your plots and those of Herring, the data showed systematic disagreement in the early data (seen as "dips" or gaps) with your fits. Have you and Herring looked into this discrepancy?

Reply by Herring: The anomaly in obliquity in earlier 1981 has been noted and we are investigating these data. These data do not significantly affect the corrections to the nutation series coefficients.

Dickey: You mentioned that your data span is really too short to determine a good value for the 18.6-year nutation term. It should be stressed that besides the long-term 18.6-year nutation factor, there is a linear term due to possible precession constant correction that must be considered also.

Kaplan: I noticed you solved for the 13.7-day term. There's also a much smaller, 13.6-day term. One doesn't expect a significant correction to it, but in a limited data set - especially if you're solving for both orthogonal components - there might be "leakage" of correction estimates from one term to the other.

Reply by Herring: Yes, we simultaneously estimated the 13.66-day and 13.63-day periods. The correction still was to the (larger) 13.66-day term.

Eubanks: It seems that your daily nutation estimates from before 1984 are much less scattered than the results that Doug Robertson presented. Can you explain this?

Reply by Himwich: Our software and solutions are essentially the same as what Doug used but there could be differences in the details of the parameterization. 